\title{
Robotic CT-guided out-of-plane needle insertion: comparison of angle accuracy with manual insertion in phantom and measurement of distance accuracy in animals
}

\author{
Toshiyuki Komaki $^{1} \cdot$ Takao Hiraki $^{1}$ (D) Tetsushi Kamegawa ${ }^{2} \cdot$ Takayuki Matsuno $^{3} \cdot$ Jun Sakurai $^{4} \cdot$ Ryutaro Matsuura $^{5}$. \\ Takuya Yamaguchi $^{6} \cdot$ Takanori Sasaki $^{7}$. Toshiharu Mitsuhashi ${ }^{4}$. Soichiro Okamoto ${ }^{1} \cdot$ Mayu Uka ${ }^{1}$. Yusuke Matsui ${ }^{1}$. \\ Toshihiro Iguchi $^{1} \cdot$ Hideo Gobara ${ }^{8} \cdot$ Susumu Kanazawa ${ }^{1}$
}

Received: 16 July 2019 / Revised: 30 August 2019 / Accepted: 27 September 2019 / Published online: 26 November 2019

(C) The Author(s) 2019

\begin{abstract}
Objectives To evaluate the accuracy of robotic CT-guided out-of-plane needle insertion in phantom and animal experiments. Methods A robotic system (Zerobot), developed at our institution, was used for needle insertion. In the phantom experiment, 12 robotic needle insertions into a phantom at various angles in the $X Y$ and $Y Z$ planes were performed, and the same insertions were manually performed freehand, as well as guided by a smartphone application (SmartPuncture). Angle errors were compared between the robotic and smartphone-guided manual insertions using Student's $t$ test. In the animal experiment, 6 robotic out-ofplane needle insertions toward targets of $1.0 \mathrm{~mm}$ in diameter placed in the kidneys and hip muscles of swine were performed, each with and without adjustment of needle orientation based on reconstructed CT images during insertion. Distance accuracy was calculated as the distance between the needle tip and the target center.

Results In the phantom experiment, the mean angle errors of the robotic, freehand manual, and smartphone-guided manual insertions were $0.4^{\circ}, 7.0^{\circ}$, and $3.7^{\circ}$ in the $X Y$ plane and $0.6^{\circ}, 6.3^{\circ}$, and $0.6^{\circ}$ in the $Y Z$ plane, respectively. Robotic insertions in the $X Y$ plane were significantly $(p<0.001)$ more accurate than smartphone-guided insertions. In the animal experiment, the overall mean distance accuracy of robotic insertions with and without adjustment of needle orientation was $2.5 \mathrm{~mm}$ and $5.0 \mathrm{~mm}$, respectively.

Conclusion Robotic CT-guided out-of-plane needle insertions were more accurate than smartphone-guided manual insertions in the phantom and were also accurate in the in vivo procedure, particularly with adjustment during insertion.

Key Points

- Out-of-plane needle insertions performed using our robot were more accurate than smartphone-guided manual insertions in the phantom experiment and were also accurate in the in vivo procedure.

- In the phantom experiment, the mean angle errors of the robotic and smartphone-guided manual out-of-plane needle insertions were $0.4^{\circ}$ and $3.7^{\circ}$ in the XY plane $(p<0.001)$ and $0.6^{\circ}$ and $0.6^{\circ}$ in the YZ plane $(p=0.65)$, respectively.
\end{abstract}

Electronic supplementary material The online version of this article (https://doi.org/10.1007/s00330-019-06477-1) contains supplementary material, which is available to authorized users.

Takao Hiraki

hiraki-t@cc.okayama-u.ac.jp

1 Department of Radiology, Okayama University Medical School, 2-5-1 Shikatacho, Kitaku, Okayama 700-8558, Japan

2 Graduate School of Interdisciplinary Science and Engineering in Health Systems, Okayama University, 3-1-1 Tsushimanaka, Kitaku, Okayama 700-8530, Japan

3 Graduate School of Natural Science and Technology, Okayama University, 3-1-1 Tsushimanaka, Kitaku, Okayama 700-8530, Japan

4 Center for Innovative Clinical Medicine, Okayama University Hospital, 2-5-1 Shikatacho, Kitaku, Okayama 700-8558, Japan
5 Graduate School of Health Sciences, Okayama University Medical School, 2-5-1 Shikatacho, Kitaku, Okayama 700-8558, Japan

6 Division of Radiology, Department of Medical Technology, Okayama University Hospital, 2-5-1 Shikatacho, Kitaku, Okayama 700-8558, Japan

Collaborative Research Center for OMIC, Okayama University Graduate School of Medicine, Dentistry, and Pharmaceutical Sciences, 2-5-1 Shikatacho, Kitaku, Okayama 700-8558, Japan

8 Division of Medical Informatics, Okayama University Hospital, 2-5-1 Shikatacho, Kitaku, Okayama 700-8558, Japan 
- In the animal experiment, the overall mean distance accuracies of the robotic out-of-plane needle insertions with and without adjustments of needle orientation during insertion were $2.5 \mathrm{~mm}$ and $5.0 \mathrm{~mm}$, respectively.

Keywords Robotics $\cdot$ Interventional radiology $\cdot$ Animal experiments

\section{Abbreviation \\ CT Computed tomography}

\section{Introduction}

Computed tomography (CT)-guided interventional procedures such as ablation and biopsy primarily comprise needle insertion into the lesion under $\mathrm{CT}$ guidance. Although needle insertion is generally performed in the axial CT plane (i.e., inplane insertion), out-of-plane needle insertion is occasionally required to achieve an anatomically safer tract. For example, needle insertion into the hepatic dome of the liver and the renal upper pole along a craniocaudally oblique tract may avoid transthoracic insertion accompanied by risks of pneumothorax and hemothorax $[1,2]$. However, such needle insertions with freehand manual techniques are generally challenging because it is difficult to set a needle at the angle required and to maintain it as such during insertion [3]. Further, adjustment of needle orientation based on $\mathrm{CT}$ images during insertion is also difficult, because the entire needle and the target are not observed in the same two-dimensional CT plane. Additionally, target movement due to respiration may make it even more difficult. Although multiplanar reconstructions may help confirm needle orientation, they require additional time and expertise.

We have been developing a robotic system to enable CTguided needle insertion [4-6]. In our previous studies, we found accurate robotic in-plane insertions of various types of needles in animal experiments $[4,5]$. However, robotic out-ofplane insertions had not yet been evaluated. Unlike manual insertion, the robot allows users to set the needle at an exact predetermined angle before insertion and the needle posture during robotic insertion may be more stable. Thus, we hypothesized that accurate out-of-plane needle insertion could be achieved with the use of the robot. The aim of the present study is, therefore, to evaluate the accuracy of robotic outof-plane needle insertion in phantom and animal experiments.

\section{Materials and methods}

The animal experiment was approved by the institutional animal care and use committee of our institution. The design of the robotic system (Zerobot, Medicalnet Okayama) used herein (Fig. 1) has been described previously [4-6]. Briefly, the system was designed by the authors (T.H., T. Kamegawa, and
T. Matsuno) at Okayama University and then manufactured by Medicalnet Okayama. The robot has 6 degrees of freedom, and its tasks are to hold, locate, orient, and insert a needle under CT guidance. The needle is attached to a needle holder at the end of a robot arm. The robot may be manipulated by either button operation of the controller or numerical inputs on software displayed on the touch panel. With the former technique, the robot moves while the buttons of the controller are manually pressed, whereas with the latter technique, the robot moves to a certain place semi-automatically after numerical inputs. We had no role with the company that manufactured the robot.

\section{Phantom experiment}

The phantom experiment was designed to evaluate the angle accuracy of robotic needle insertion, freehand manual insertion, and manual insertion guided by a smartphone (iPhone 6 ,

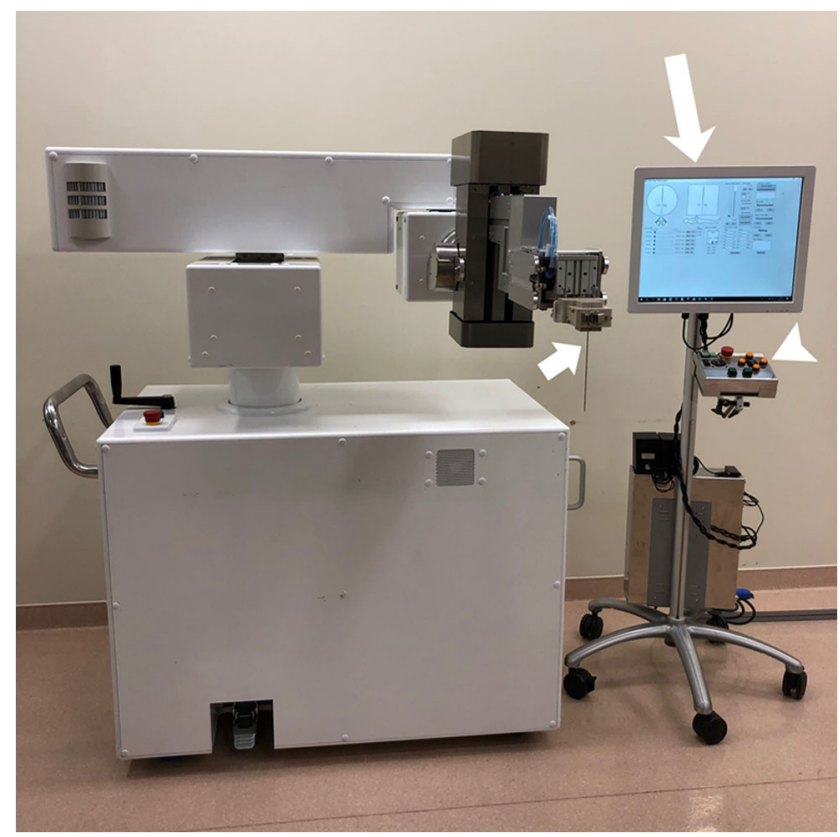

Fig. 1 The robotic system. A robot (left) with 6 degrees of freedom and an interface (right) comprising a touch panel (long arrow) and a controller (arrowhead). The robot may be manipulated by either button operation of the controller or numerical inputs on software displayed on the touch panel. With the former technique, the robot moves while the buttons of the controller are manually pressed, whereas with the latter technique, the robot moves to a certain place semi-automatically after numerical inputs. In the present study, typically, alteration of needle angles for needle targeting and adjustment of needle orientation was performed by the latter, while needle insertion was done by the former. The needle (short arrow) is attached to a plastic needle holder at the end of a robot arm 
Apple Inc.) application (SmartPuncture, Matsuyama Shimin Hospital). The endpoint was the angle errors in the $X Y$ and $Y Z$ planes (i.e., axial and sagittal CT planes, respectively). A 17 gauge biopsy introducer needle (TASK Laboratory) with six combinations of angles (Table 1) was inserted to a depth of approximately $8 \mathrm{~cm}$ into a melamine sponge $[7,8]$ fixed on the CT table (Fig. 2). For robotic insertions, the operator (T. Komaki, who had a 5-year experience in CT-guided interventions and a 3-year experience in manipulation of the robot) operated the robot to set the needle at predetermined angles, followed by insertion. In the manual group, freehand insertions (i.e., without guiding tools) were performed first. Then, the smartphone-guided manual insertions were performed with similar techniques as previously described [7]. Briefly, a planned angle in the $X Y$ plane was entered on the application. Then, a guideline with the angle was displayed on the screen. The tilted angle number of the smartphone against the direction of gravity, which corresponded to the angle in the $Y Z$ plane, was also displayed on the screen. The operator inserted the needle manually along the guideline while holding the smartphone with the planned angle in the $Y Z$ plane (Supplementary Fig. S1). Those manual insertions were performed by two operators (Y.M. and M.U. with 12-year and 11year experiences, respectively, in CT-guided interventions). Needle insertion time was measured. CT scanning (Aquilion 64, Canon Medical Systems) was performed after each insertion.

The needle angle was measured in maximum intensity projections in the two planes at a CT console by a blinded author (S.O. with a 3-year experience in CT-guided interventions). The angle error was calculated as the difference between the predetermined angle and the angle after insertion. Then, the

Table 1 Needle Angles for Insertion in the Phantom Experiment

Needle angles $\left({ }^{\circ}\right)$ No. of insertions

\begin{tabular}{|c|c|c|c|c|c|c|}
\hline \multirow[b]{3}{*}{$\begin{array}{l}\text { XY } \\
\text { plane }\end{array}$} & \multirow[b]{3}{*}{$\begin{array}{l}\text { YZ } \\
\text { plane }\end{array}$} & \multirow[t]{3}{*}{ Robotic } & \multicolumn{4}{|l|}{ Manual } \\
\hline & & & \multicolumn{2}{|c|}{ Smartphone-guided } & \multicolumn{2}{|l|}{ Freehand } \\
\hline & & & $\begin{array}{l}\text { Operator } \\
1\end{array}$ & $\begin{array}{l}\text { Operator } \\
2\end{array}$ & $\begin{array}{l}\text { Operator } \\
1\end{array}$ & $\begin{array}{l}\text { Operator } \\
2\end{array}$ \\
\hline 40 & -20 & 2 & 1 & 1 & 1 & 1 \\
\hline 30 & -30 & 2 & 1 & 1 & 1 & 1 \\
\hline 20 & -40 & 2 & 1 & 1 & 1 & 1 \\
\hline-20 & 20 & 2 & 1 & 1 & 1 & 1 \\
\hline-30 & 30 & 2 & 1 & 1 & 1 & 1 \\
\hline-40 & 40 & 2 & 1 & 1 & 1 & 1 \\
\hline
\end{tabular}

The needle angle is defined as the angle between the perpendicular line and the needle on CT images. When the needle is oriented to the right and left side of the perpendicular line, the needle angle is expressed as a positive and negative value, respectively

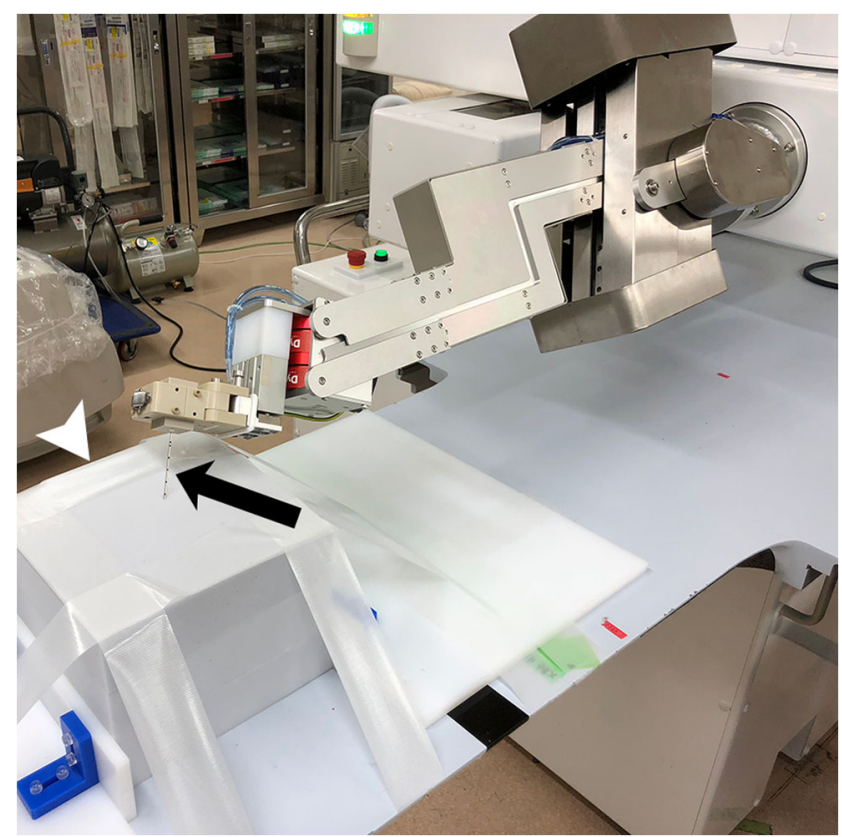

Fig. 2 Robotic out-of-plane needle insertion in the phantom experiment. The needle (arrow) at the predetermined angles in the $X Y$ and $Y Z$ planes is inserted into a sponge phantom (arrowhead) fixed on the CT table

angle errors were compared between the robotic and smartphone-guided manual insertions. Using angle errors, the three-dimensional deviations of the needle tip at a depth of $8 \mathrm{~cm}$ were estimated (detailed calculation methods are provided in Appendix 1 in the Supplementary Material).

\section{Animal experiment}

The animal experiment was performed to evaluate the in vivo distance accuracy of robotic out-of-plane insertion. The 17gauge biopsy introducer needle (TASK Laboratory) was used for insertion in two female swine (weight, $56.7 \mathrm{~kg}$ and 54.3 $\mathrm{kg}$ ) (management techniques are provided in Appendix 2 in the Supplementary Material). The hip muscle and the kidney were selected as locations for robotic insertion, as they allow for safe and adequately long out-of-plane needle tracts and evaluation of insertions both with and without respiratory motion. Before the experiment, 1.0-mm-diameter tungsten balls (Humanity) were placed into the locations as targets using an 18-gauge coaxial needle manually inserted with in-plane CT guidance. Depths of the targets from the skin ranged from 64.7 to $83.3 \mathrm{~mm}$.

First, six insertions were made with three combinations of needle angles each into the hip muscle and the kidney (Table 2). These were performed without adjustments of the needle orientation during insertion, in order to evaluate the in vivo accuracy of methods similar to those adopted in the phantom experiment. Then, the same insertions were performed with adjustments during insertion, in order to confirm improved accuracy with adjustments. Insertions into the kidney were performed 
Table 2 Needle Angles for Insertion in the Animal Experiment

\begin{tabular}{rlllll}
\hline Needle angles $\left(^{\circ}\right)$ & \multicolumn{2}{l}{ No. of insertions } \\
\cline { 3 - 6 } & & \multicolumn{2}{l}{ With adjustments } & Without adjustments \\
\hline XY plane & YZ plane & Hip muscle & Kidney & Hip muscle & Kidney \\
$+30 \pm 15$ & $-30 \pm 10$ & 2 & 2 & 2 & 2 \\
$0 \pm 15$ & $-30 \pm 10$ & 2 & 2 & 2 & 2 \\
$-30 \pm 15$ & $-30 \pm 10$ & 2 & 2 & 2 & 2 \\
\hline
\end{tabular}

The needle angle is defined as the angle between the perpendicular line and the needle on CT images. When the needle is oriented to the right and left side of the perpendicular line, the needle angle is expressed as a positive and negative value, respectively

with breath hold by the ventilator, while insertions into the hip muscle were performed without breath hold.

The robot was manipulated by the same author (T. Komaki) in the phantom experiment. CT scanning (Eminence STARGATE, Shimadzu Inc.) was performed to determine the starting point for insertion, the needle angles required, and the needle tract length. Subsequently, the needle was set with the angles and then its tip was moved to the starting point. Correct needle orientation was confirmed by CT scanning along with reconstructed three-dimensional CT images. The needle was then inserted till the whole tract length at once in the group without adjustment. In the group with adjustment, in contrast, the needle orientation was checked by CT scans at two time points (Fig. 3). The needle orientation was corrected as necessary, based on deviations between the ideal and actual needle angles and needle behavior during alteration of needle angles in vivo (Fig. 4). In both groups, needle insertion time, radiation exposure (i.e., tube current-time product and dose-length product) to the swine during insertion, and the number of CT scans and needle adjustments during insertion were recorded. CT scanning was performed after insertion.

Distance accuracy was evaluated on axial CT images of $0.5 \mathrm{~mm}$ thickness with $0.3-\mathrm{mm}$ intervals using software (OsiriX, version 5.7.1, OsiriX Foundation) by an author (S.O.). First, whether the needle hit the target was evaluated on the reconstructed three-dimensional images. The distance accuracy was then determined by calculating the Euclidean distance between the target center and the needle tip, using the CT coordinates. Lateral and depth errors were further evaluated (Appendix 3 in the Supplementary Material). The actual length of needle tract and the amount of target movement during insertion (i.e., the distance between the target centers before and after insertion) were calculated, using the CT coordinates.

\section{Statistical analysis}

In the phantom experiment, the number of needle insertions was calculated to verify the superiority of the robotic insertion

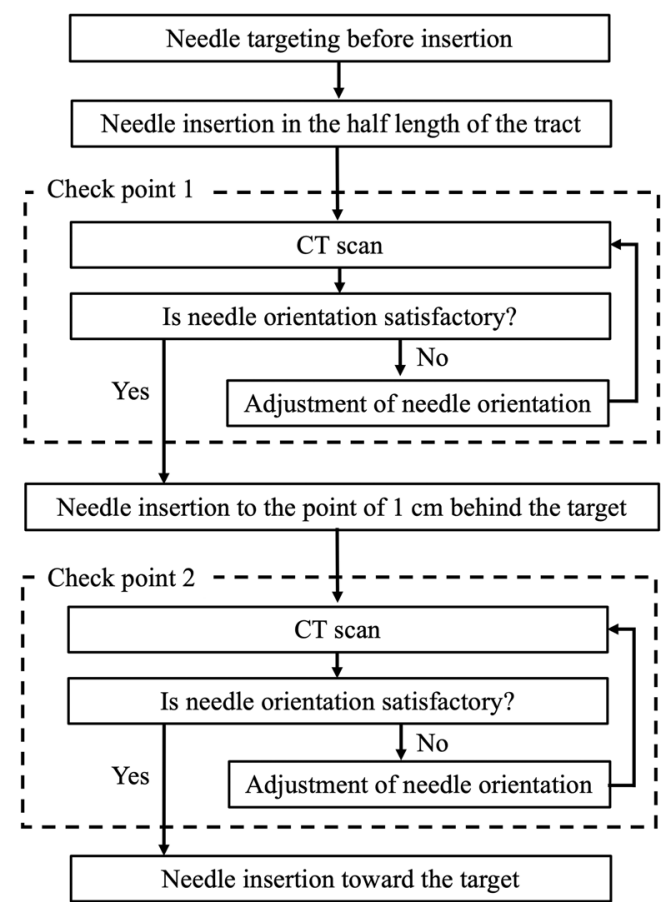

Fig. 3 Techniques of needle insertion with adjustment in the animal experiment. Needle orientation is checked by CT scanning at two points: middle of the tract and $1 \mathrm{~cm}$ behind the target. Needle orientation is then evaluated in maximum intensity projections reconstructed from $\mathrm{CT}$ data in the $X Y$ and $Y Z$ planes. If the needle orientation is not satisfactory, it is adjusted until it becomes satisfactory

compared to smartphone-guided manual insertion with respect to the angle accuracy. The absolute value of the mean difference in the angle errors between the two groups was estimated to be $1.0^{\circ}$, with a standard deviation of $0.5^{\circ}$ in both

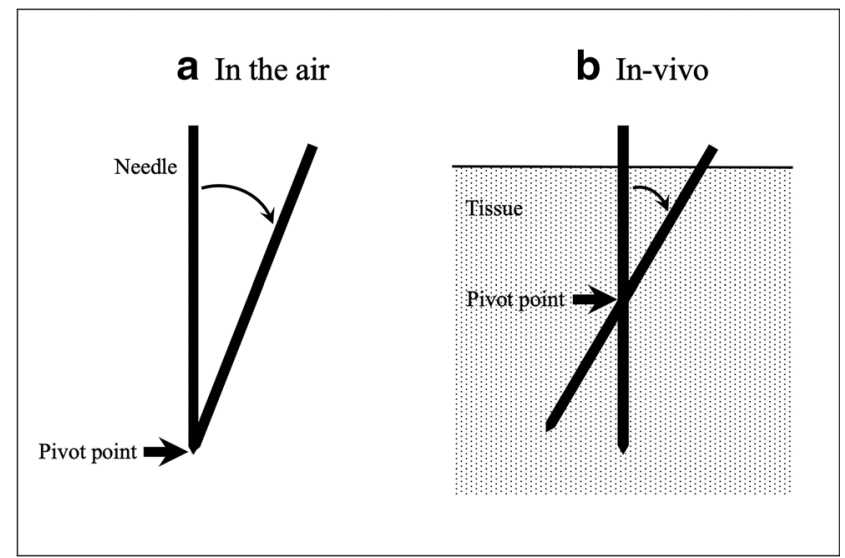

Fig. 4 Schemata of the needle angle being changed by the robot in the air (a) and in vivo (b). The robot provides the remote-center-of-motion function, by which the needle angle is changed around its tip in the air. In vivo, however, the needle angle is changed as if a pivot point is at the approximately half length of needle in the tissue; this is attributable mainly to resistance of the tissue. Considering this characteristic needle behavior in vivo, the corrected needle angle in the planes may be calculated to compensate for the deviation between the ideal and actual needle angles 
groups. The correlation coefficient between the two planes (i.e., the $X Y$ and $Y Z$ planes) was hypothesized to be $0.3^{\circ}$. With an $\alpha$ value of 0.05 and a $\beta$ value of $0.2,12$ insertions in each group were required.

Numerical variables were compared using a two-sided Student's $t$ test. A $p$ value of $<0.05$ was considered statistically significant. Statistical analysis was performed by an author (S.O.) using EZR software (EZR, version 1.33, Saitama Medical Center).

\section{Results}

\section{Phantom experiment}

The results of the phantom experiment are summarized in Table 3. The mean angle errors of the robotic, freehand manual, and smartphone-guided manual insertions were $0.4^{\circ}$ (range, $0.0-1.6^{\circ}$ ), $7.0^{\circ}$ (range, $1.0-17.1^{\circ}$ ), and $3.7^{\circ}$ (range, $0.0-7.4^{\circ}$ ) in the $X Y$ plane and $0.6^{\circ}$ (range, $0.0-1.2^{\circ}$ ), $6.3^{\circ}$ (range, $1.8-12.1^{\circ}$ ), and $0.6^{\circ}$ (range, $0.1-1.5^{\circ}$ ) in the $Y Z$ plane, respectively. Robotic insertions were significantly $(p<0.001)$ more accurate than smartphone-guided manual insertions in the $X Y$ plane. Robotic insertions resulted in significantly $(p<0.001)$ smaller predicted needle tip deviations (mean, $1.0 \mathrm{~mm}$; range, $0.1-2.5 \mathrm{~mm}$ ) than did smartphone-guided manual insertions (mean, $4.9 \mathrm{~mm}$; range, $0.9-9.7 \mathrm{~mm}$ ) at the insertion depth of $8 \mathrm{~cm}$. Robotic insertions were significantly $(p<0.001)$ faster (mean, $5.0 \mathrm{~s}$; range, 4.8-5.1 s) than smartphone-guided manual insertions (mean, $24.7 \mathrm{~s}$; range, $13.8-43.3 \mathrm{~s})$.

\section{Animal experiment}

The results of the animal experiment are summarized in Table 4. The mean tract length was $82.2 \mathrm{~mm}$ (range, 71.1$87.5 \mathrm{~mm}$ ) and $82.5 \mathrm{~mm}$ (range, $69.9-92.0 \mathrm{~mm}$ ) in the groups with and without adjustments, respectively. The needle appeared to hit the target in 4 of the 12 insertions without adjustment and in 11 of the 12 insertions with adjustment. The mean distance accuracy of the robotic needle insertions with adjustment was $2.5 \mathrm{~mm}$ (range, $0.9-3.8 \mathrm{~mm}$ ) in the hip muscle and $2.4 \mathrm{~mm}$ (range, 1.9-3.3 $\mathrm{mm}$ ) in the kidney, while that of robotic needle insertions without adjustment was $5.1 \mathrm{~mm}$ (range, 3.7-6.6 mm) in the hip muscle and $5.0 \mathrm{~mm}$ (range, $2.7-8.3 \mathrm{~mm}$ ) in the kidney. Overall distance accuracy with adjustment (mean, $2.5 \mathrm{~mm}$; range, 0.9-3.8 mm) was significantly $(p<0.001)$ better than that without adjustment (mean, 5.0 ; range, $2.7-8.3 \mathrm{~mm}$ ). The results of lateral and depth errors are shown in Supplementary Table S1. Time for needle insertions with adjustment (mean, $716.9 \mathrm{~s}$; range, 316-1851 s) was significantly $(p<0.001)$ longer than that without adjustment (mean, $14.4 \mathrm{~s}$; range, 11-19 s), requiring the median of four CT scans and the median of two needle adjustments during insertion. The tube current-time product (mean, $7210.1 \mathrm{mAs}$; range, 3999-14,533 $\mathrm{mAs}$ ) and dose-length product (mean, $998.3 \mathrm{mGy} \cdot \mathrm{cm}$; range, 553.8-2011.7 $\mathrm{mGy} \cdot \mathrm{cm}$ ) during insertion in the group with adjustments were significantly ( $p<0.001$ for both) larger than the tube current-time product (mean, $1310.8 \mathrm{mAs}$; range, 1066-1333 $\mathrm{mAs}$ ) and doselength product (mean, $181.5 \mathrm{mGy} \cdot \mathrm{cm}$; range, 147.7-184.6 $\mathrm{mGy} \cdot \mathrm{cm}$ ) during insertion in the group without adjustments.

\section{Discussion}

The present study was conducted to evaluate the accuracy of out-of-plane needle insertion using our robot; such an insertion is generally difficult to perform accurately by hand. The results showed that our robot achieved accurate out-of-plane needle insertions in the phantom and the animals, which may indicate more choices for selection of needle trajectories in clinical cases, possibly making the procedure safer and more effective. In particular, it is notable that junior staff and even

Table 3 Results of the phantom experiment

\begin{tabular}{lllr}
\hline & Robotic & Manual & $p$ value ${ }^{\mathrm{a}}$ \\
\cline { 3 - 4 } & & Smartphone-guided & Freehand \\
\hline $\begin{array}{l}\text { Needle insertion time (s) } \\
\text { Needle insertion accuracy }\left(^{\circ}\right)\end{array}$ & $5.0 \pm 0.1(4.8-5.1)$ & $24.7 \pm 8.1(13.8-43.3)$ & $4.4 \pm 1.8(3.0-9.4)$ \\
$\begin{array}{l}X Y \text { plane } \\
Y Z \text { plane }\end{array}$ & $0.4 \pm 0.4(0.0-1.6)$ & $3.7 \pm 2.3(0.0-7.4)$ & $7.0 \pm 5.7(1.0-17.1)$ \\
$\begin{array}{l}\text { Predicted needle tip deviation at } \\
\text { a depth of } 8 \mathrm{~cm}(\mathrm{~mm})\end{array}$ & $0.6 \pm 0.4(0.0-1.2)$ & $0.6 \pm 0.4(0.1-1.5)$ & $6.3 \pm 3.5(1.8-12.1)$ \\
\hline
\end{tabular}

Data are means \pm standard deviations, with ranges in parentheses

${ }^{a}$ Comparison between robotic and manual smartphone-guided insertions with Student's $t$ test 
Table 4 Results of the animal experiment

\begin{tabular}{|c|c|c|c|}
\hline & \multicolumn{2}{|l|}{ Robotic insertion } & \multirow[t]{2}{*}{$p$ value } \\
\hline & With adjustment & Without adjustment & \\
\hline Needle tract length $(\mathrm{mm})^{\mathrm{a}}$ & $82.2 \pm 5.3(71.1-87.5)$ & $82.5 \pm 8.0(69.9-92.0)$ & 0.903 \\
\hline No. of needle adjustments during insertion ${ }^{\mathrm{b}}$ & $2(0-5)$ & $0(0-0)$ & $<0.001$ \\
\hline No. of CT scans during insertion ${ }^{\mathrm{b}}$ & $4(2-8)$ & $0(0-0)$ & $<0.001$ \\
\hline Needle insertion time $(\mathrm{s})^{\mathrm{a}}$ & $716.9 \pm 396.0(316-1851)$ & $14.4 \pm 2.6(11-19)$ & $<0.001$ \\
\hline Distance of target movement $(\mathrm{mm})^{\mathrm{a}}$ & $4.6 \pm 2.5(1.2-11.2)$ & $4.8 \pm 0.8(3.8-6.6)$ & 0.776 \\
\hline \multicolumn{4}{|l|}{ Radiation exposure to swine during insertion } \\
\hline Tube current-time product $(\mathrm{mAs})^{\mathrm{a}}$ & $7210.1 \pm 2746.4(3999-14,533)$ & $1310.8 \pm 77.1(1066-1333)$ & $<0.001$ \\
\hline Dose-length product $(\mathrm{mGy} \cdot \mathrm{cm})^{\mathrm{a}}$ & $998.3 \pm 380.1(553.8-2011.7)$ & $181.5 \pm 10.7(147.7-184.6)$ & $<0.001$ \\
\hline \multicolumn{4}{|l|}{ Needle insertion accuracy $(\mathrm{mm})$} \\
\hline Hip muscle $(n=6)^{\mathrm{a}}$ & $2.5 \pm 1.0(0.9-3.8)$ & $5.1 \pm 1.2(3.7-6.6)$ & 0.003 \\
\hline Kidney $(n=6)^{\mathrm{a}}$ & $2.4 \pm 0.5(1.9-3.3)$ & $5.0 \pm 2.2(2.7-8.3)$ & 0.019 \\
\hline Total $(n=12)^{\mathrm{a}}$ & $2.5 \pm 0.8(0.9-3.8)$ & $5.0 \pm 1.7(2.7-8.3)$ & $<0.001$ \\
\hline
\end{tabular}

residents may perform out-of-plane insertion with difficult trajectories using our robot.

Smartphone applications to assist needle insertion such as SmartPuncture and OncoGuide (National Institutes of Health) seem quite unique [7, 9]. A phantom study [7] indicated that the mean angle errors of smartphone-guided needle insertion were $<1.8^{\circ}$ in the $X Y$ plane and $<4.1^{\circ}$ in the $Y Z$ plane. The phantom experiment in the current study revealed that robotic out-of-plane needle insertion was significantly more accurate than smartphone-guided manual insertion. The advantages of the robot are that the needle angles required may be easily and accurately obtained by numerical inputs on the touch panel and the angles may be maintained during insertion.

Despite accurate needle angles in the phantom, the distance accuracy of robotic insertions in animals was limited to some extent if the needle orientation was not adjusted during insertion. This was attributed mainly to movement of targets and needle deviation during insertion $[4,5]$. Although the distance accuracy of insertions without adjustment up to $8.3 \mathrm{~mm}$ might be acceptable for some interventional procedures (e.g., biopsy for lesions of $\geq 17 \mathrm{~mm}$ in diameter), more accurate insertion is usually required for lesions that are small and/or make contact with at-risk structures. To improve the accuracy, we adopted two check points to correct needle orientation. Needle deflection, which was more likely to occur by tissue displacement (especially the skin and subcutaneous tissue) during insertion, was mainly compensated at the half point of the tract. Target movement, which was more likely to occur when the needle tip was close to the target, was mainly compensated at the point of $1 \mathrm{~cm}$ behind the target. The two-step needle adjustment during insertion significantly improved the accuracy to a mean value of $2.5 \mathrm{~mm}$. Such distance accuracy seems comparable to that of in-plane insertion with this robot in previous animal experiments $[4,5]$. Notably, however, insertions with adjustment required greater radiation exposure as well as more time. Therefore, the necessity and appropriate number for needle adjustments should be determined individually based on the accuracy required in the case.

Other than our robot, several devices and robots to assist out-of-plane needle insertion have been evaluated. An electromagnetically guided system (IMACTIS) displays the needle path in real time on two-dimensional CT images reconstructed from preprocedural CT data [3, 10]. A phantom study [3] reported a median distance accuracy of $3.7 \mathrm{~mm}$ in out-ofplane trajectories using this navigation system. In addition, a prospective randomized clinical trial [10] demonstrated that the needle insertion accuracy was significantly improved with this navigation system, when compared to insertion with conventional CT guidance (median distance accuracy, $4.1 \mathrm{~mm}$ vs. $8.9 \mathrm{~mm}$ ) in various clinical conditions including out-of-plane trajectory. Some robotic positioning systems have been commercialized, including iSYS (Kitzbuhel) [8, 11-13] and MAXIO (Perfint Healthcare) [14-17]. Unlike our robotic system, the task of those robots is confined to needle targeting (i.e., orientation of the needle) based on preprocedural CT data. Therefore, needle insertion must be manually performed by physicians. Accuracy of needle insertion including out-ofplane trajectory with these systems has been evaluated [8, 11-17]. For example, an animal study using MAXIO [15] demonstrated that the mean distance accuracy was $4.7 \mathrm{~mm}$, which seems comparable to the insertion accuracy without adjustment in our animal experiment. The abovementioned electromagnetically guided system and robotic positioning systems are based on preprocedural CT data and therefore 
do not allow for a response to intraprocedural positional alteration (e.g., target movement and the patient's body motion) and needle deviation. On the other hand, our robot enables response to intraprocedural alteration, which has the potential to improve insertion accuracy.

There were some limitations to our study. The type of the needle used, locations for needle insertion in swine, and combinations of needle angles tested were limited. Further, techniques of breath hold with the ventilator employed in the animal experiments were different from those in conscious patients. Therefore, it remains to be confirmed whether similar results can be obtained with other needles and angles at other locations in conscious clinical cases; this is an area requiring future research

In conclusion, robotic CT-guided out-of-plane needle insertions were more accurate than smartphone-guided manual insertions in the phantom and were also quite accurate in the in vivo procedure, particularly with adjustment of needle orientation during insertion.

Acknowledgments The authors thank Keiji Tanimoto for his expertise to develop the robot and Masaaki Hirata (Department of Radiology, Matsuyama Shimin Hospital) for the pieces of advice to the use of smartphone application (SmartPuncture).

Funding information This study has received funding by a grant from the Japan Society for the Promotion of Science (JSPS) (17K10439). A part of the robot used in this study was provided by Cannon Medical Systems. The project on the robot was financially supported by grants from the Promotion of Science and Technology, Okayama Prefecture; the Japan Agency for Medical Research and Development (AMED) (15hk0102014h001, 15hk0102014h002, 16hk0102014h003); the Society for the Promotion of Science (JSPS) (25461882, 18K07677); the Organization for Research Promotion and Collaboration, Okayama University; Bayer research grant, Japan; Radiology Society; Cannon Medical Systems; and Chugoku Regional Innovation Research Center. The funders' sources had no role in the study design; in the data collection, analysis, and interpretation; in the writing of the report; or in the decision to submit the article for publication.

\section{Compliance with ethical standards}

Guarantor The scientific guarantor of this publication is Dr. Takao Hiraki.

Conflict of interest Dr. Takao Hiraki and Dr. Susumu Kanazawa declare relationships with Cannon Medical Systems. No other authors of this manuscript declare relationships with any companies.

Statistics and biometry One of the authors has significant statistical expertise.

Informed consent Approval from the institutional animal care committee was obtained.

Ethical approval Institutional review board approval was not required because this study was not performed on human subjects. The animal experiment was approved by the institutional animal care and use committee of our institution.

\section{Methodology}

- prospective

- experimental

- performed at one institution

Open Access This article is distributed under the terms of the Creative Commons Attribution 4.0 International License (http:// creativecommons.org/licenses/by/4.0/), which permits unrestricted use, distribution, and reproduction in any medium, provided you give appropriate credit to the original author(s) and the source, provide a link to the Creative Commons license, and indicate if changes were made.

\section{References}

1. Shibata T, limuro Y, Yamamoto Y et al (2002) CT-guided transthoracic percutaneous ethanol injection for hepatocellular carcinoma not detectable with US. Radiology 223:115-120

2. Shibata T, Shibata T, Maetani Y et al (2004) Transthoracic percutaneous radiofrequency ablation for liver tumors in the hepatic dome. J Vasc Interv Radiol 15:1323-1327

3. Moncharmont L, Moreau-Gaudry A, Medici M, Bricault I (2015) Phantom evaluation of a navigation system for out-of-plane CTguided puncture. Diagn Interv Imaging 96:531-536

4. Hiraki T, Kamegawa T, Matsuno T, Komaki T, Sakurai J, Kanazawa S (2017) Robotically driven CT-guided needle insertion: preliminary results in phantom and animal experiments. Radiology 285:454-461

5. Hiraki T, Matsuno T, Kamegawa T et al (2018) Robotic insertion of various ablation needles under computed tomography guidance: accuracy in animal experiments. Eur J Radiol 105:162-167

6. Hiraki T, Kamegawa T, Matsuno T et al (2018) Zerobot ${ }^{\circledR}$ : a remotecontrolled robot for needle insertion in CT-guided interventional radiology developed at Okayama University. Acta Med Okayama 72:539-546

7. Hirata M, Watanabe R, Koyano Y et al (2017) Using a motion sensor-equipped smartphone to facilitate CT-guided puncture. Cardiovasc Intervent Radiol 40:609-615

8. Schulz B, Eichler K, Siebenhandl P et al (2013) Accuracy and speed of robotic assisted needle interventions using a modern cone beam computed tomography intervention suite: a phantom study. Eur Radiol 23:198-204

9. Xu S, Krishnasamy V, Levy E, Li M, Tse ZTH, Wood BJ (2018) Smartphone-guided needle angle selection during CT-guided procedures. AJR Am J Roentgenol 210:207-213

10. Durand P, Moreau-Gaudry A, Silvent AS et al (2017) Computer assisted electromagnetic navigation improves accuracy in computed tomography guided interventions: a prospective randomized clinical trial. PLoS One 12:1-19

11. Kettenbach J, Kara L, Toporek G, Fuerst M, Kronreif G (2014) A robotic needle-positioning and guidance system for CT-guided puncture: ex vivo results. Minim Invasive Ther Allied Technol 23:271-278

12. Groetz S, Wilhelm K, Willinek W, Pieper C, Schild H, Thomas D (2016) A new robotic assistance system for percutaneous CTguided punctures: initial experience. Minim Invasive Ther Allied Technol 25:79-85

13. Engstrand J, Toporek G, Harbut P, Jonas E, Nilsson H, Freedman J (2017) Stereotactic CT-guided percutaneous microwave ablation of liver tumors with the use of high-frequency jet ventilation: an accuracy and procedural safety study. AJR Am J Roentgenol 208: 193-200 
14. Abdullah BJ, Yeong CH, Goh KL et al (2015) Robotic-assisted thermal ablation of liver tumours. Eur Radiol 25:246-257

15. Cornelis F, Takaki H, Laskhmanan M et al (2015) Comparison of CT fluoroscopy-guided manual and CT-guided robotic positioning system for in vivo needle placements in swine liver. Cardiovasc Intervent Radiol 38:1252-1260

16. Koethe Y, Xu S, Velusamy G, Wood BJ, Venkatesan AM (2014) Accuracy and efficacy of percutaneous biopsy and ablation using robotic assistance under computed tomography guidance: a phantom study. Eur Radiol 24:723-730
17. Smakic A, Rathmann N, Kostrzewa M, Schönberg SO, Weiß C, Diehl SJ (2018) Performance of a robotic assistance device in computed tomography-guided percutaneous diagnostic and therapeutic procedures. Cardiovasc Intervent Radiol 41:639-644

Publisher's note Springer Nature remains neutral with regard to jurisdictional claims in published maps and institutional affiliations. 